TITLE:

\title{
Construction of the least informative observable conserved by a given quantum instrument
}

$\operatorname{AUTHOR}(\mathrm{S})$ :

Kuramochi, Yui

\section{CITATION:}

Kuramochi, Yui. Construction of the least informative observable conserved by a given quantum instrument. Journal of Mathematical Physics 2015, 56(9): 092202.

\section{ISSUE DATE:}

2015-09-28

URL:

http://hdl.handle.net/2433/203028

\section{RIGHT:}

(c) 2015 AIP Publishing LLC. This article may be downloaded for

personal use only. Any other use requires prior permission of the author and the American Institute of Physics. 


\section{AIP $\left.\right|_{\text { Joumal of }}$ Mathematical Physics}

Construction of the least informative observable conserved by a given quantum instrument

Yui Kuramochi

Citation: Journal of Mathematical Physics 56, 092202 (2015); doi: 10.1063/1.4931625

View online: http://dx.doi.org/10.1063/1.4931625

View Table of Contents: http://scitation.aip.org/content/aip/journal/jmp/56/9?ver=pdfcov

Published by the AIP Publishing

\section{Articles you may be interested in}

Quantum Information

Am. J. Phys. 78, 654 (2010); 10.1119/1.3302835

Energy conservation in quantum mechanics

Am. J. Phys. 72, 580 (2004); 10.1119/1.1648326

Quantum Computation and Quantum Information

Am. J. Phys. 70, 558 (2002); 10.1119/1.1463744

Conservation of information: Quantum mechanics and RIS

AIP Conf. Proc. 584, 87 (2001); 10.1063/1.1405588

The qualitative theory of elementary transformations of one- and multichannel quantum systems in the inverse-problem approach. The construction of transformations with given spectral parameters Phys. Part. Nucl. 30, 111 (1999); 10.1134/1.953100

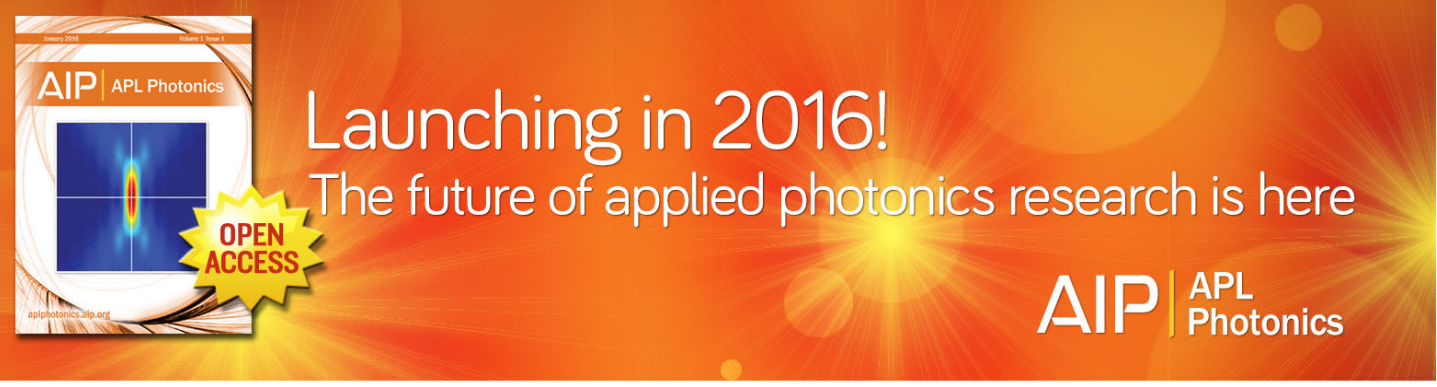




\title{
Construction of the least informative observable conserved by a given quantum instrument
}

\author{
Yui Kuramochia) \\ Department of Nuclear Engineering, Kyoto University, 6158540 Kyoto, Japan
}

(Received 27 May 2015; accepted 11 September 2015; published online 28 September 2015)

\begin{abstract}
For a quantum measurement process described by a quantum instrument $\mathcal{I}$ and a system observable corresponding to a positive-operator valued measure (POVM) $E, I$ is said to conserve the information of $E$ if the joint successive measurement of $\mathcal{I}$ followed by $E$ is equivalent to a single measurement of $E$. We show that for any quantum instrument $\mathcal{I}$ we can construct a POVM conserved by $\mathcal{I}$. Intuitively, the construction gives the infinite joint successive measurement of $\mathcal{I}$. We also show that the constructed POVM is the least informative observable among POVMs conserved by $\mathcal{I}$, i.e., the constructed POVM can be realized by a classical post-processing of any POVM conserved by $\mathcal{I}$. As typical examples of quantum instruments, we explicitly evaluate POVMs of infinite successive measurements for photon counting and quantum counter instruments. (C) 2015 AIP Publishing LLC. [http://dx.doi.org/10.1063/1.4931625]
\end{abstract}

\section{INTRODUCTION}

While quantum measurement back-action causes the state change and the information loss of the system, some kind of information is known to be conserved if we focus on a proper system observable described by a positive-operator valued measure (POVM). An example of such measurement processes is the photon counting measurement, also known as the Srinivas-Davies model, ${ }^{1}$ in which we can estimate photon number of the pre-measurement state from that of the post-measurement state and the measurement outcome. In general, a measurement process described by a quantum instrument ${ }^{2} I$ is said to conserve a system observable described by a positive-operator valued measure $E$ if the joint measurement of $\mathcal{I}$ followed by $E$ is equivalent to a single measurement of $E$.

In the previous work, ${ }^{3}$ the author examined some physical examples of quantum instruments and showed that some intuitively "natural" observable for each instrument satisfies the conservation condition. Then, it is natural to ask whether there exists a POVM $E$ conserved by a given quantum instrument $\mathcal{I}$. The answer, the main result of this paper, is affirmative, and $E$ can be constructed as the infinite successive joint measurement of $\mathcal{I}$, called an infinite composition of $\mathcal{I}$. Furthermore, this infinite successive measurement is shown to be characterized by the minimality up to the fuzzy preorder relation ${ }^{4-7}$ among the conserved POVMs, i.e., the infinite successive measurement is the least informative POVM conserved by $\mathcal{I}$. We also reconsider the photon counting and quantum counter instruments and explicitly derive their infinite compositions. As a by-product of the discus-

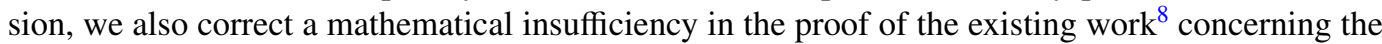
convergence of the normalized count number in the quantum counter measurement.

In Ref. 3, the conservation condition was introduced as a sufficient condition for the "relativeentropy conservation law" for system observable $E$, which is the generalization of "Shannon entropy conservation" derived by Ban..$^{9}$ In the comparison of two POVMs, the discussion of Ref. 3 is largely based on the concept of the sufficient statistic, ${ }^{10}$ while it can be formulated more rigorously and briefly by using the fuzzy preorder and equivalence relations between POVMs $^{4-7}$ (Definition

a)Electronic mail: kuramochi.yui.22c@st.kyoto-u.ac.jp 
1). The reformulation of the information conservation condition based on the fuzzy equivalence relation is another purpose of the present paper.

This paper is organized as follows. In Sec. II, some preliminary results concerning the fuzzy preorder and equivalence relations and the composition of quantum measurement processes are reviewed. In Sec. III, we construct the infinite composition of a given quantum instrument with a standard Borel outcome space and show that it is the least informative POVM that is conserved by the instrument. In Sec. IV, we consider photon counting and quantum counter instruments and derive the explicit forms of their infinite compositions. Sec. V summarizes the main results of this paper.

\section{PRELIMINARIES}

In this section we briefly review some preliminary results on the quantum theory of measurement and fix the notation. For a general reference of quantum measurement, we refer Refs. 5, 11, and 12 .

\section{A. Positive-operator valued measures and fuzzy preorder and equivalence relations}

We fix a complex Hilbert space $\mathcal{H}$ and denote the set of bounded linear operators on $\mathcal{H}$ as $\mathcal{L}(\mathcal{H})$. Let $(\Omega, \mathscr{B})$ be a measurable space. A mapping $E: \mathscr{B} \rightarrow \mathcal{L}(\mathcal{H})$ is called a POVM with its outcome space $(\Omega, \mathscr{B})$ if

(i) $E(B) \geq O(\forall B \in \mathscr{B})$,

(ii) $E(\Omega)=I$,

(iii) for any disjoint $\left\{B_{i}\right\}_{i \geq 1} \subset \mathscr{B}, E\left(\cup_{i \geq 1} B_{i}\right)=\sum_{i \geq 1} E\left(B_{i}\right)$ in the weak operator topology.

Here $O$ and $I$ are zero and identity operators, respectively. Let $E$ be a POVM with its outcome space $(\Omega, \mathscr{B})$. A measurable set $N \in \mathscr{B}$ is called an $E$-null set if $E(N)=O$. $E$-almost sure equations and convergences for stochastic variables are also defined in a similar manner as for a classical probability measure.

Let $\left(\Omega_{1}, \mathscr{B}_{1}\right)$ and $\left(\Omega_{2}, \mathscr{B}_{2}\right)$ be measurable spaces. A mapping $v .(\cdot): \Omega_{2} \times \mathscr{B}_{1} \ni\left(\omega_{2}, B\right) \mapsto v_{\omega_{2}}(B)$ $\in[0,1]$ is said to be a Markov kernel if

(i) for each $\omega_{2} \in \Omega_{2}$, the mapping $v_{\omega_{2}}(\cdot): \mathscr{B}_{1} \rightarrow[0,1]$ is a probability measure,

(ii) for each $B \in \mathscr{B}_{1}$, the mapping $v .(B): \Omega_{2} \rightarrow[0,1]$ is $\mathscr{B}_{2}$-measurable.

Let $E^{1}$ and $E^{2}$ be POVMs with their outcome spaces $\left(\Omega_{1}, \mathscr{B}_{1}\right)$ and $\left(\Omega_{2}, \mathscr{B}_{2}\right)$, respectively. Following Refs. 5-7, we define a relation $E^{1} \leq E^{2}$ by the existence of a Markov kernel $v(\cdot): \Omega_{2} \times \mathscr{B}_{1} \rightarrow[0,1]$ such that

$$
E^{1}(B)=\int_{\Omega_{2}} v_{\omega_{2}}(B) E^{2}\left(d \omega_{2}\right) \quad\left(\forall B \in \mathscr{B}_{1}\right),
$$

and we say that $E^{1}$ is fuzzier than $E^{2}$. POVMs $E^{1}$ and $E^{2}$ are said to be equivalent, denoted as $E^{1} \simeq E^{2}$, if $E^{1} \leq E^{2}$ and $E^{2} \leq E^{1}$. The relations $\leq$ and $\simeq$ are preorder and equivalence relations for POVMs,${ }^{6,7}$ respectively. Equation (1) intuitively means that the measurement of $E^{1}$ can be realized by performing the measurement of $E^{2}$ and the classical information processing on the measurement outcome $\omega_{2}$.

The following lemma concerning the Markov kernel will be used later.

Lemma 1. 1. Let $\left(\Omega_{i}, \mathscr{B}_{i}\right)(i=1,2,3)$ be measurable spaces. Suppose that

$$
\begin{aligned}
v_{.}^{1}(\cdot): \Omega_{2} \times \mathscr{B}_{1} & \rightarrow[0,1], \\
v^{2}(\cdot): \Omega_{3} \times \mathscr{B}_{2} & \rightarrow[0,1]
\end{aligned}
$$

are Markov kernels. Then $v^{3}(\cdot)$ defined by

$$
v_{\omega_{3}}^{3}(B):=\int_{\Omega_{2}} v_{\omega_{2}}^{1}(B) v_{\omega_{3}}^{2}\left(d \omega_{2}\right) \quad\left(B \in \mathscr{B}_{1}, \omega_{3} \in \Omega_{3}\right)
$$

is a Markov kernel. 
2. Let $\left(\Omega_{i}, \mathscr{B}_{i}\right)(i=1,2,3)$ be measurable spaces and let $v \cdot(\cdot): \Omega_{3} \times \mathscr{B}_{2} \rightarrow[0,1]$ be a Markov kernel. Define $\tilde{v} .(\cdot)$ by

$$
\begin{gathered}
\tilde{v}_{\left(\omega_{1}, \omega_{3}\right)}(B):=v_{\omega_{3}}\left(\left.B\right|_{\omega_{1}}\right), \\
\left.B\right|_{\omega_{1}}:=\left\{\omega_{2} \in \Omega_{2} \mid\left(\omega_{1}, \omega_{2}\right) \in B\right\}
\end{gathered}
$$

for $\left(\omega_{1}, \omega_{3}\right) \in \Omega_{1} \times \Omega_{3}$ and $B \in \mathscr{B}_{1} \times \mathscr{B}_{2}$. Then $\tilde{v} .(\cdot)$ is a Markov kernel.

Proof. 1 is shown in the proof of Proposition 1 in Ref. 6. We show 2. For each $\left(\omega_{1}, \omega_{3}\right) \in$ $\Omega_{1} \times \Omega_{3}, \tilde{v}_{\left(\omega_{1}, \omega_{3}\right)}(\cdot)$ is a probability measure. To show the measurability of $\tilde{v}$. $(B)\left(B \in \mathscr{B}_{1} \times \mathscr{B}_{2}\right)$, define a class $\mathscr{D}$ of subsets of $\Omega_{1} \times \Omega_{2}$ by

$$
\mathscr{D}:=\left\{B \in \mathscr{B}_{1} \times \mathscr{B}_{2} \mid \tilde{v} .(B) \text { is } \mathscr{B}_{1} \times \mathscr{B}_{3} \text {-measurable }\right\} .
$$

Then $\mathscr{D}$ is a Dynkin system ( $\lambda$-system), i.e., it is closed under countable disjoint unions and proper differences and contains $\Omega_{1} \times \Omega_{2}$. For each $B_{i} \in \mathscr{B}_{i}(i=1,2)$,

$$
\tilde{v}_{\left(\omega_{1}, \omega_{3}\right)}\left(B_{1} \times B_{2}\right)=\chi_{B_{1}}\left(\omega_{1}\right) v_{\omega_{3}}\left(B_{2}\right)
$$

is $\mathscr{B}_{1} \times \mathscr{B}_{3}$-measurable, where $\chi_{B}(\cdot)$ is an indicator function for a subset $B$. Thus, $\mathscr{D}$ contains the class of the cylinder sets $\left\{B_{1} \times B_{2} \mid B_{1} \in \mathscr{B}_{1}, B_{2} \in \mathscr{B}_{2}\right\}$ and the Dynkin's theorem assures that $\mathscr{D}$ coincides with $\mathscr{B}_{1} \times \mathscr{B}_{2}$, which proves the assertion.

\section{B. Completely positive instruments and their compositions}

Let $(\Omega, \mathscr{B})$ be a measurable space. A completely positive $(C P)$ instrument $^{2,12,13}$ (in the Heisenberg picture) with its outcome space $(\Omega, \mathscr{B})$ is a mapping

$$
\mathcal{I} .(\cdot): \mathscr{B} \times \mathcal{L}(\mathcal{H}) \ni(B, a) \mapsto \mathcal{I}_{B}(a) \in \mathcal{L}(\mathcal{H})
$$

such that

(i) for any $B \in \mathscr{B}, \mathcal{I}_{B}(\cdot): \mathcal{L}(\mathcal{H}) \rightarrow \mathcal{L}(\mathcal{H})$ is a normal CP linear map,

(ii) for any disjoint $\left\{B_{i}\right\}_{i \geq 1} \subset \mathscr{B}$ and any $a \in \mathcal{L}(\mathcal{H}), \mathcal{I}_{\cup_{i \geq 1} B_{i}}(a)=\sum_{i \geq 1} \mathcal{I}_{B_{i}}(a)$ in the ultraweak operator topology,

(iii) $\mathcal{I}_{\Omega}(I)=I$.

We also define a positive $(\mathrm{P})$ instrument by replacing $\mathrm{CP}$ with $\mathrm{P}$ in the definition of the $\mathrm{CP}$ instrument. A CP instrument $\mathcal{I}(\cdot(\cdot)$ with its outcome space $(\Omega, \mathscr{B})$ describes the statistics of the measurement outcome and the state change due to the measurement simultaneously in a necessary and sufficient manner. The POVM corresponding to the probability distribution of the measurement outcome is given by $E(B)=\mathcal{I}_{B}(I)(B \in \mathscr{B})$. Here we only consider the case when the range and the domain of $\mathcal{I}_{B}(\cdot)$ are identical, which is a necessary condition so that we can compare the same observable of the system before and after the measurement.

A measurable space $(\Omega, \mathscr{B})$ Borel isomorphic to a complete separable metric space is called a standard Borel space. ${ }^{14} \mathrm{~A}(\mathrm{C}) \mathrm{P}$ instrument (respectively, POVM) with a standard Borel outcome space is called a standard Borel (C)P instrument (respectively, a standard Borel POVM). In the rest of this paper, we only consider standard Borel (C)P instruments and POVMs.

The following theorem, which is a slight modification of the theorem due to Davies and Lewis, ${ }^{12,13}$ assures the existence of a CP instrument and a POVM corresponding to a joint successive measurement process.

Theorem 1. (i) Let $\mathcal{I}^{i}(\cdot)$ be a CP instrument with a standard Borel outcome space $\left(\Omega_{i}, \mathscr{B}_{i}\right)$ $(i=1,2)$. Then there exists a unique $C P$ instrument $\mathcal{I}^{12}(\cdot)$ with the product outcome space $\left(\Omega_{1} \times \Omega_{2}, \mathscr{B}_{1} \times \mathscr{B}_{2}\right)$ such that

$$
\mathcal{I}_{B_{1} \times B_{2}}^{12}(\cdot)=\mathcal{I}_{B_{1}}^{1} \circ \mathcal{I}_{B_{2}}^{2}(\cdot) \quad\left(B_{1} \in \mathscr{B}_{1}, B_{2} \in \mathscr{B}_{2}\right) .
$$

(ii) Let $\mathcal{I}^{1}(\cdot)$ be a CP instrument with a standard Borel outcome space $\left(\Omega_{1}, \mathscr{B}_{1}\right)$ and let $E^{2}$ be a POVM with a standard Borel outcome space $\left(\Omega_{2}, \mathscr{B}_{2}\right)$. Then there exists a unique POVM $E^{12}$ 
with the product outcome space $\left(\Omega_{1} \times \Omega_{2}, \mathscr{B}_{1} \times \mathscr{B}_{2}\right)$ such that

$$
E^{12}\left(B_{1} \times B_{2}\right)=\mathcal{I}_{B_{1}}^{1}\left(E^{2}\left(B_{2}\right)\right) \quad\left(B_{1} \in \mathscr{B}_{1}, B_{2} \in \mathscr{B}_{2}\right) .
$$

We call the CP instrument $\mathcal{I}^{12}(\cdot)$ and the POVM $E^{12}$ as compositions, and denote them as $\left(\mathcal{I}^{1} * \mathcal{I}^{2}\right) \cdot(\cdot)$ and $\mathcal{I}^{1} * E^{2}$, respectively.

Proof. We first show (i). According to Theorem 4.2.2 of Ref. 12, there exists a unique P instrument $\mathcal{I}^{12}(\cdot)$ such that condition (3) holds. To show the complete positivity of $\mathcal{I}^{12}(\cdot)$, define a class $\mathscr{D}$ of subsets of $\Omega_{1} \times \Omega_{2}$ by

$$
\mathscr{D}:=\left\{B \in \mathscr{B}_{1} \times \mathscr{B}_{2} \mid \mathcal{I}_{B}^{12}(\cdot) \text { is } \mathrm{CP}\right\} .
$$

From condition (3), $\mathscr{D}$ contains the class of cylinder sets. Since we can easily verify that $\mathscr{D}$ is a Dynkin class, Dynkin's theorem assures that $\mathscr{D}=\mathscr{B}_{1} \times \mathscr{B}_{2}$, proving assertion (i).

To show (ii), take a CP instrument $\mathcal{I}^{2}(\cdot)$ with the outcome space $\left(\Omega_{2}, \mathscr{B}_{2}\right)$ such that $\mathcal{I}_{B}^{2}(I)=$ $E^{2}(B)\left(B \in \mathscr{B}_{2}\right)$. Then a POVM $E^{12}(\cdot):=\left(\mathcal{I}^{1} * \mathcal{I}^{2}\right) .(I)$ satisfies condition (4). The uniqueness can be shown by using the Dynkin's theorem as parallel as in the classical measure.

Now let $\mathcal{I}^{k}(\cdot)$ be a CP instrument with a standard Borel outcome space $\left(\Omega_{k}, \mathscr{B}_{k}\right)(k=1,2, \ldots)$ and let $E$ be a POVM with a standard Borel outcome space $(\Omega, \mathscr{B})$. Then we have the following associative laws for the composition:

$$
\begin{aligned}
\left(\mathcal{I}^{1} * \mathcal{I}^{2}\right) * \mathcal{I}^{3} & =\mathcal{I}^{1} *\left(\mathcal{I}^{2} * \mathcal{I}^{3}\right), \\
\left(\mathcal{I}^{1} * \mathcal{I}^{2}\right) * E & =\mathcal{I}^{1} *\left(\mathcal{I}^{2} * E\right) .
\end{aligned}
$$

Thus, we may write these CP instrument and POVM as $\mathcal{I}^{1} * \mathcal{I}^{2} * \mathcal{I}^{3}$ and $\mathcal{I}^{1} * \mathcal{I}^{2} * E$, respectively. Multiple compositions $\mathcal{I}^{1} * \cdots * \mathcal{I}^{n}$ and $\mathcal{I}^{1} * \cdots * \mathcal{I}^{n} * E$ for general $n \geq 1$ are also defined in a similar manner. These are the unique CP instrument and POVM such that

$$
\begin{gathered}
\left(\mathcal{I}^{1} * \cdots * \mathcal{I}^{n}\right)_{B_{1} \times \cdots \times B_{n}}(\cdot)=\mathcal{I}_{B_{1}}^{1} \circ \cdots \circ \mathcal{I}_{B_{n}}^{n}(\cdot) \\
\left(\mathcal{I}^{1} * \cdots * \mathcal{I}^{n} * E\right)\left(B_{1} \times \cdots \times B_{n} \times B\right)=\mathcal{I}_{B_{1}}^{1} \circ \cdots \circ \mathcal{I}_{B_{n}}^{n}(E(B))
\end{gathered}
$$

for each $B_{k} \in \mathscr{B}_{k}(k=1, \ldots, n)$ and $B \in \mathscr{B}$.

For later use, we show the following lemmas.

Lemma 2. Let $\mathcal{I}^{1}(\cdot)$ be a CP instrument with a standard Borel outcome space $\left(\Omega_{1}, \mathscr{B}_{1}\right)$ and let $E^{2}$ be a POVM with a standard Borel outcome space $\left(\Omega_{2}, \mathscr{B}_{2}\right)$. Then for each $B_{1} \in \mathscr{B}_{1}$ and for each bounded complex valued $\mathscr{B}_{2}$-measurable function $f$,

$$
\int_{\Omega_{1} \times \Omega_{2}} \chi_{B_{1}}\left(\omega_{1}\right) f\left(\omega_{2}\right)\left(\mathcal{I}^{1} * E^{2}\right)\left(d \omega_{1} \times d \omega_{2}\right)=\mathcal{I}_{B_{1}}^{1}\left(\int_{\Omega_{2}} f\left(\omega_{2}\right) E^{2}\left(d \omega_{2}\right)\right) .
$$

Proof. It is sufficient to show Eq. (5) when $f \geq 0$. When $f$ is a measurable simple function, Eq. (5) holds. For general $f$, take a monotone sequence of non-negative measurable simple functions $f_{n}$ such that $f_{n}\left(\omega_{2}\right) \uparrow f\left(\omega_{2}\right)$ for each $\omega_{2} \in \Omega_{2}$. Then from the dominated convergence theorem we have

$$
\begin{gathered}
\int_{\Omega_{1} \times \Omega_{2}} \chi_{B_{1}}\left(\omega_{1}\right) f_{n}\left(\omega_{2}\right)\left(\mathcal{I}^{1} * E^{2}\right)\left(d \omega_{1} \times d \omega_{2}\right) \uparrow \int_{\Omega_{1} \times \Omega_{2}} \chi_{B_{1}}\left(\omega_{1}\right) f\left(\omega_{2}\right)\left(\mathcal{I}^{1} * E^{2}\right)\left(d \omega_{1} \times d \omega_{2}\right), \\
\int_{\Omega_{2}} f_{n}\left(\omega_{2}\right) E^{2}\left(d \omega_{2}\right) \uparrow \int_{\Omega_{2}} f\left(\omega_{2}\right) E^{2}\left(d \omega_{2}\right) .
\end{gathered}
$$

Since $\mathcal{I}_{B_{1}}^{1}(\cdot)$ is normal, Eq. (7) implies that

$$
\mathcal{I}_{B_{1}}^{1}\left(\int_{\Omega_{2}} f_{n}\left(\omega_{2}\right) E^{2}\left(d \omega_{2}\right)\right) \uparrow \mathcal{I}_{B_{1}}^{1}\left(\int_{\Omega_{2}} f\left(\omega_{2}\right) E^{2}\left(d \omega_{2}\right)\right) .
$$

Since LHSs of Eqs. (6) and (8) coincide, we obtain Eq. (5). 
Lemma 3. Let $\mathcal{I}^{1}(\cdot)$ be a CP instrument with a standard Borel outcome space $\left(\Omega_{1}, \mathscr{B}_{1}\right)$ and let $E^{2}$ and $E^{3}$ be POVMs with standard Borel outcome spaces $\left(\Omega_{2}, \mathscr{B}_{2}\right)$ and $\left(\Omega_{3}, \mathscr{B}_{3}\right)$, respectively. Then we have

(i) if $E^{2} \leq E^{3}$, then $\mathcal{I}^{1} * E^{2} \leq \mathcal{I}^{1} * E^{3}$,

(ii) if $E^{2} \simeq E^{3}$, then $\mathcal{I}^{1} * E^{2} \simeq \mathcal{I}^{1} * E^{3}$.

Proof. We first show (i). From the assumption $E^{2} \leq E^{3}$, there exists a Markov kernel $\nu .(\cdot): \Omega_{3}$ $\times \mathscr{B}_{2} \rightarrow[0,1]$ such that

$$
E^{2}\left(B_{2}\right)=\int_{\Omega_{3}} v_{\omega_{3}}\left(B_{2}\right) E^{3}\left(d \omega_{3}\right) \quad\left(B_{2} \in \mathscr{B}_{2}\right) .
$$

From Lemma 1, we can define a POVM $E^{12}$ by

$$
E^{12}(B):=\int_{\Omega_{1} \times \Omega_{3}} v_{\omega_{3}}\left(\left.B\right|_{\omega_{1}}\right)\left(\mathcal{I}^{1} * E^{3}\right)\left(d \omega_{1} \times d \omega_{3}\right) \quad\left(B \in \mathscr{B}_{1} \times \mathscr{B}_{2}\right) .
$$

From the definition of $E^{12}, E^{12} \leq \mathcal{I}^{1} * E^{3}$ holds. On the other hand, for each $B_{1} \in \mathscr{B}_{1}$ and $B_{2} \in \mathscr{B}_{2}$, we have

$$
\begin{aligned}
E^{12}\left(B_{1} \times B_{2}\right) & =\int_{\Omega_{1} \times \Omega_{3}} \chi_{B_{1}}\left(\omega_{1}\right) v_{\omega_{3}}\left(B_{2}\right)\left(\mathcal{I}^{1} * E^{3}\right)\left(d \omega_{1} \times d \omega_{3}\right) \\
& =\mathcal{I}_{B_{1}}^{1}\left(\int_{\Omega_{3}} v_{\omega_{3}}\left(B_{2}\right) E^{3}\left(d \omega_{3}\right)\right) \\
& =\mathcal{I}_{B_{1}}^{1}\left(E^{2}\left(B_{2}\right)\right)=\left(\mathcal{I}^{1} * E^{2}\right)\left(B_{1} \times B_{2}\right),
\end{aligned}
$$

where we have used Lemma 2 in the derivation of the second equality. Therefore, we obtain $\mathcal{I}^{1} * E^{2}=E^{12} \leq \mathcal{I}^{1} * E^{3}$ and (i) is proved. (ii) immediately follows from (i) and the definition of the equivalence relation $\simeq$.

\section{INFINITE COMPOSITION OF AN INSTRUMENT AND ITS MINIMAL INFORMATION-CONSERVING PROPERTY}

Definition 1. Let I.(.) be a standard Borel CP instrument and let $E$ be a standard Borel POVM. We say that $E$ is conserved by $\mathcal{I}$, or $\mathcal{I}$ conserves $E$, if $\mathcal{I} * E \simeq E$.

This condition is essentially the same as the one obtained in the author's previous work ${ }^{3}$ for a sufficient condition for the "relative-entropy conservation law" which is a generalization of the "Shannon-entropy conservation law" derived by Ban. ${ }^{9}$

From Definition 1 and Lemma 3, we immediately obtain the following theorem.

Theorem 2. Let I.(.) be a standard Borel CP instrument and let $E^{1}$ and $E^{2}$ be standard Borel POVMs. Suppose that $E^{1} \simeq E^{2}$. Then $\mathcal{I} .(\cdot)$ conserves $E^{1}$ if and only if $\mathcal{I}(\cdot \cdot)$ conserves $E^{2}$. In other words, the conservation by I.(.) is well-defined to $\simeq$-equivalence classes of standard Borel POVMs.

Now, we ask whether there exists a standard Borel POVM $E$ that is conserved by a given standard Borel CP instrument $\mathcal{I}(\cdot(\cdot)$. The answer is affirmative, and it is given by a POVM called the infinite composition of $\mathcal{I}$ corresponding to an infinite successive measurement of $\mathcal{I}$. The conservation of the infinite composition $E_{\infty}$ by a CP instrument $\mathcal{I}$ is intuitively understood as follows: the composition $\mathcal{I} * E_{\infty}$ is a measurement process in which we first perform $\mathcal{I}$ and then perform $\mathcal{I}$ infinitely many times, which is obviously equivalent to performing $E_{\infty}$. Furthermore, we will show in Theorem 4 that the infinite composition $E_{\infty}$ of $\mathcal{I}$ is special to $\mathcal{I}$ in the sense that $E_{\infty}$ is the minimal element with respect to the preorder relation $\leq$ among POVMs conserved by $\mathcal{I}$.

The following proposition due to Tumulka ${ }^{15}$ is a key to the construction of the infinite composition, which is a POVM version of the celebrated Kolmogorov extension theorem for probability measures. ${ }^{16}$ 
Proposition 1 (quantum Kolmogorov extension theorem). Let $\left(\Omega_{i}, \mathscr{B}_{i}\right)(i=1,2, \ldots)$ be a standard Borel space and let $E_{n}(n=1,2, \ldots)$ be a POVM with the product outcome space $\left(\prod_{i=1}^{n} \Omega_{i}\right.$, $\left.\prod_{i=1}^{n} \mathscr{B}_{i}\right)$. Suppose that $\left\{E_{n}\right\}$ satisfies the condition

$$
E_{n}(B)=E_{n+1}\left(B \times \Omega_{n+1}\right) \quad\left(n \geq 1, B \in \prod_{i=1}^{n} \mathscr{B}_{i}\right) .
$$

Then, there exists a unique POVM $E_{\infty}$ with the infinite product outcome space $\left(\prod_{i=1}^{\infty} \Omega_{i}, \prod_{i=1}^{\infty} \mathscr{B}_{i}\right)$ such that

$$
E_{n}(B)=E_{\infty}\left(B \times \prod_{i=n+1}^{\infty} \Omega_{i}\right) \quad\left(n \geq 1, B \in \prod_{i=1}^{n} \mathscr{B}_{i}\right) .
$$

Condition (9) is called a Kolmogorov consistency condition and the POVM $E_{\infty}$ satisfying (10) is said to be consistent with $\left\{E_{n}\right\}$. Note that the consistent POVM $E_{\infty}$ is a standard Borel POVM since a countable product of standard Borel spaces is also a standard Borel space.

For simplicity, if $\left(\Omega_{i}, \mathscr{B}_{i}\right)(1 \leq i \leq n)$ is identical to $(\Omega, \mathscr{B})$, the product space $\left(\prod_{i=1}^{n} \Omega_{i}, \prod_{i=1}^{n}\right.$ $\left.\mathscr{B}_{i}\right)(1 \leq n \leq \infty)$ is denoted as $\left(\Omega^{n}, \mathscr{B}^{n}\right)$. We also denote $\underbrace{\mathcal{I} * \cdots * \mathcal{I}}_{n \text { elements }}$ as $\mathcal{I}^{* n}$ for a $\mathrm{CP}$ instrument $\mathcal{I}$.

Now we construct the infinite composition of a $\mathrm{CP}$ instrument.

Theorem 3 (infinite composition of an instrument). Let I.(·) be a CP instrument with a standard Borel outcome space $(\Omega, \mathscr{B})$. Then there exists a unique POVM $E_{\infty}$ with the infinite product outcome space $\left(\Omega^{\infty}, \mathscr{B}^{\infty}\right)$ such that

$$
E_{\infty}\left(\prod_{i=1}^{n} B_{i} \times \Omega^{\infty}\right)=\mathcal{I}_{B_{1}} \circ \cdots \circ \mathcal{I}_{B_{n}}(I)
$$

for each $n \geq 1$ and $B_{i} \in \mathscr{B}(i=1, \ldots, n)$. The POVM $E_{\infty}$ is called an infinite composition of $\mathcal{I}$.

Proof. Let us define a POVM $E_{n}(\cdot):=\left(\mathcal{I}^{* n}\right) .(I)$ for each $n \geq 1$. Since $E_{n}\left(\prod_{i=1}^{n} B_{i}\right)$ coincides with the RHS of Eq. (11), from the quantum Kolmogorov extension theorem, it is sufficient to show that $\left\{E_{n}\right\}$ satisfies the Kolmogorov consistency condition. For each $B \in \mathscr{B}^{n}(n \geq 1)$, we have

$$
\begin{aligned}
E_{n+1}(B \times \Omega) & =\left(\mathcal{I}^{* n} * \mathcal{I}\right)_{B \times \Omega}(I) \\
& =\mathcal{I}_{B}^{* n}\left(\mathcal{I}_{\Omega}(I)\right) \\
& =\mathcal{I}_{B}^{* n}(I)=E_{n}(B),
\end{aligned}
$$

and the theorem holds.

The next theorem is the main result of this paper, which states that for a given CP instrument $\mathcal{I}$, the infinite composition of $\mathcal{I}$ is the least informative POVM conserved by $\mathcal{I}$.

Theorem 4. Let I. (.) be a CP instrument with a standard Borel outcome space $(\Omega, \mathscr{B})$ and let $E_{\infty}$ be the infinite composition of $\mathcal{I}$. Then, $\mathcal{I}$ conserves $E_{\infty}$. Furthermore, $E_{\infty}$ is the minimal element with respect to the preorder relation $\leq$ among the standard Borel POVMs conserved by $\mathcal{I}$.

Proof. $\left(\Omega^{\infty}, \mathscr{B}^{\infty}\right)$ and $\left(\Omega \times \Omega^{\infty}, \mathscr{B} \times \mathscr{B}^{\infty}\right)$ are Borel isomorphic by the mapping

$$
\Omega \times \Omega^{\infty} \ni\left(\omega, \prod_{i=1}^{\infty} \omega_{i}\right) \mapsto\left(\omega, \omega_{1}, \omega_{2}, \ldots\right) \in \Omega^{\infty},
$$

and we consider $\mathcal{I} * E_{\infty}$ as a POVM with the outcome space $\left(\Omega^{\infty}, \mathscr{B}^{\infty}\right)$ by this identification. Then for each $B, B_{1}, \ldots, B_{n} \in \mathscr{B}(1 \leq n<\infty)$, we have

$$
\begin{aligned}
\left(\mathcal{I} * E_{\infty}\right)\left(B \times B_{1} \times \cdots B_{n} \times \Omega^{\infty}\right) & =\mathcal{I}_{B}\left(E_{\infty}\left(B_{1} \times \cdots B_{n} \times \Omega^{\infty}\right)\right) \\
& =\mathcal{I}_{B} \circ \mathcal{I}_{B_{1}} \circ \cdots \circ \mathcal{I}_{B_{n}}(I) \\
& =E_{\infty}\left(B \times B_{1} \times \cdots B_{n} \times \Omega^{\infty}\right) .
\end{aligned}
$$


From the uniqueness of $E_{\infty}$, we obtain $\mathcal{I} * E_{\infty}=E_{\infty}$, which proves the conservation of $E_{\infty}$ by I.

To show the minimality of $E_{\infty}$, take an arbitrary POVM $F$ with a standard Borel outcome space $\left(\Omega_{X}, \mathscr{B}_{X}\right)$ such that $F \simeq \mathcal{I} * F$. The goal of the proof is to construct a Markov kernel corresponding to an information-procession from $\Omega_{X}$ to $\Omega^{\infty}$. Since $\mathcal{I} * F \leq F$, there exists a Markov kernel $\tilde{v}^{1}(\cdot): \Omega_{X} \times\left(\mathscr{B} \times \mathscr{B}_{X}\right) \rightarrow[0,1]$ such that

$$
(\mathcal{I} * F)(B)=\int_{\Omega_{X}} \tilde{v}_{x}^{1}(B) F(d x)
$$

for each $B \in \mathscr{B} \times \mathscr{B}_{X}$. Since the Markov kernel $\tilde{v}_{x}^{1}\left(d \omega_{1} \times d x_{1}\right)$ corresponds to a classical information processing generating a measurement outcome $\left(\omega_{1}, x_{1}\right)$ of $\mathcal{I} * F$ from a given measurement outcome $x$ of $F$, we can construct a new Markov kernel $\tilde{v}_{x}^{2}\left(d \omega_{1} \times d \omega_{2} \times d x_{2}\right)$ which generates the measurement outcome of $\mathcal{I} * \mathcal{I} * F$ by applying the same classical information processing $\tilde{v}_{x_{1}}^{1}\left(d \omega_{2} \times d x_{2}\right)$ to $x_{1}$ which generates $\left(\omega_{2}, x_{2}\right)$. Repeating the same discussion, we can construct a sequence of Markov kernels $\tilde{v}_{x}^{n}\left(d \omega_{1} \times \cdots \times d \omega_{n} \times d x_{n}\right)$ that generates the measurement outcome of $\mathcal{I}^{* n} * F$ from that of $F$. The formal definition of the sequence $\left\{\tilde{v}^{n}(\cdot)\right\}$ is given by

$$
\tilde{v}_{x}^{n+1}(B):=\int_{\Omega^{n} \times \Omega_{X}} \tilde{v}_{x_{n}}^{1}\left(\left.B\right|_{\left.\omega^{(n)}\right)} \tilde{v}_{x}^{n}\left(d \omega^{(n)} \times d x_{n}\right)\right.
$$

for each $1 \leq n<\infty$ and each $B \in \mathscr{B}^{n+1} \times \mathscr{B}_{X}$, where we denote $\prod_{i=1}^{n} \omega_{i}$ as $\omega^{(n)}$ and $\left.B\right|_{\omega^{(n)}}:=$ $\left\{\left(\omega_{n+1}, x_{n+1}\right) \in \Omega \times \Omega_{X} \mid\left(\omega^{(n)}, \omega_{n+1}, x_{n+1}\right) \in B\right\}(1 \leq n \leq \infty)$. From Lemma 1, $\tilde{v}^{n}(\cdot)$ defined by Eq. (12) is a well-defined Markov kernel. Now we show that

$$
\left(\mathcal{I}^{* n} * F\right)(\cdot)=\int_{\Omega_{X}} \tilde{v}_{x}^{n}(\cdot) F(d x)
$$

for each $n \geq 1$. If $n=1$, Eq. (13) evident from the definition of $\tilde{v}^{1} \cdot(\cdot)$. If Eq. (13) holds for $n \geq 1$, then for each $B_{n} \in \mathscr{B}^{n}, B_{1} \in \mathscr{B}$, and $B_{X} \in \mathscr{B}_{X}$, we obtain

$$
\begin{aligned}
& \int_{\Omega_{X}} \tilde{v}_{x}^{n+1}\left(B_{n} \times B_{1} \times B^{X}\right) F(d x) \\
& =\int_{\Omega_{X}}\left(\int_{\Omega^{n} \times \Omega_{X}} \tilde{v}_{x_{n}}^{1}\left(\left.\left(B_{n} \times B_{1} \times B_{X}\right)\right|_{\left.\omega^{(n)}\right)} \tilde{v}_{x}^{n}\left(d \omega^{(n)} \times d x_{n}\right)\right) F(d x)\right. \\
& =\int_{\Omega_{X}}\left(\int_{\Omega^{n} \times \Omega_{X}} \chi_{B_{n}}\left(\omega^{(n)}\right) \tilde{v}_{x_{n}}^{1}\left(B_{1} \times B_{X}\right) \tilde{v}_{x}^{n}\left(d \omega^{(n)} \times d x_{n}\right)\right) F(d x) \\
& =\int_{\Omega^{n} \times \Omega_{X}} \chi_{B_{n}}\left(\omega^{(n)}\right) \tilde{v}_{x_{n}}^{1}\left(B_{1} \times B_{X}\right)\left(\mathcal{I}^{* n} * F\right)\left(d \omega^{(n)} \times d x_{n}\right) \\
& =\left(\mathcal{I}^{* n}\right)_{B_{n}}\left(\int_{\Omega_{X}} \tilde{v}_{x_{n}}^{1}\left(B_{1} \times B_{X}\right) F\left(d x_{n}\right)\right) \\
& =\left(\mathcal{I}^{*(n+1)} * F\right)\left(B_{n} \times B_{1} \times B_{X}\right),
\end{aligned}
$$

where in deriving Eqs. (15) and (14) we have used Lemma 2 and an equality

$$
\begin{aligned}
& \int_{\Omega_{X}}\left(\int_{\Omega^{n} \times \Omega_{X}} f\left(\omega^{(n)}, x_{n}\right) \tilde{v}_{x}^{n}\left(d \omega^{(n)} \times d x_{n}\right)\right) F(d x) \\
& =\int_{\Omega^{n} \times \Omega_{X}} f\left(\omega^{(n)}, x_{n}\right)\left(\mathcal{I}^{* n} * F\right)\left(d \omega^{(n)} \times d x_{n}\right)
\end{aligned}
$$

valid for any $\mathscr{B}^{n} \times \mathscr{B}_{X}$-measurable bounded function $f$. Equation (16) holds when $f$ is a simple function from the assumption of the induction, and for general $f$, we can prove the equation by taking a monotone sequence of simple functions converging pointwise to $f$. Thus we have shown Eq. (13)

Now we define a sequence of Markov kernels $\left\{v^{n}(\cdot)\right\}$ by

$$
v_{x}^{n}(B):=\tilde{v}_{x}^{n}\left(B \times \Omega_{X}\right) \quad\left(x \in \Omega_{X}, B \in \mathscr{B}^{n}\right) .
$$


From the definition of $\tilde{v}^{n}(\cdot)$, for each $B \in \mathscr{B}^{n}$ we have

$$
\begin{aligned}
v_{x}^{n+1}(B \times \Omega) & =\int_{\Omega^{n} \times \Omega_{X}} \tilde{v}_{x_{n}}^{1}\left(\left.\left(B \times \Omega \times \Omega_{X}\right)\right|_{\left.\omega^{(n)}\right)} \tilde{v}_{x}^{n}\left(d \omega^{(n)} \times d x_{n}\right)\right. \\
& =\int_{\Omega^{n} \times \Omega_{X}} \chi_{B}\left(\omega^{(n)}\right) \tilde{v}_{x}^{n}\left(d \omega^{(n)} \times d x_{n}\right) \\
& =\tilde{v}_{x}^{n}\left(B \times \Omega_{X}\right)=v_{x}^{n}(B) .
\end{aligned}
$$

Thus, from Kolmogorov extension theorem, there exists a probability measure $v_{x}^{\infty}(\cdot)$ with the outcome space $\left(\Omega^{\infty}, \mathscr{B}^{\infty}\right)$ such that

$$
v_{x}^{\infty}\left(B \times \Omega^{\infty}\right)=v_{x}^{n}(B) \quad\left(n \geq 1, B \in \mathscr{B}^{n}\right)
$$

for each $x \in \Omega_{X}$. To show the $\mathscr{B}_{X}$-measurability of $v_{.}^{\infty}(B)\left(B \in \mathscr{B}^{\infty}\right)$, define a class $\mathscr{D}$ of subsets of $\Omega^{\infty}$ by

$$
\mathscr{D}:=\left\{B \in \mathscr{B}^{\infty} \mid v^{\infty}(B) \text { is } \mathscr{B}_{X} \text {-measurable }\right\} .
$$

Then $\mathscr{D}$ is a Dynkin class and, from Eq. (17), $\mathscr{D}$ contains the class

$$
\left\{B \times \Omega^{\infty} \mid B \in \mathscr{B}^{n}(1 \leq n<\infty)\right\},
$$

which generates $\mathscr{B}^{\infty}$. Therefore, Dynkin's theorem assures that $\tilde{v}^{\infty}(\cdot)$ is a Markov kernel. Thus, we can define a POVM $\tilde{E}_{\infty}$ by

$$
\tilde{E}_{\infty}(B):=\int_{\Omega_{X}} v_{x}^{\infty}(B) F(d x) \quad\left(B \in \mathscr{B}^{\infty}\right),
$$

which satisfies $\tilde{E}_{\infty} \leq F$. Then for each $B \in \mathscr{B}^{n}$, we have

$$
\tilde{E}_{\infty}\left(B \times \Omega^{\infty}\right)=\int_{\Omega_{X}} \tilde{v}_{x}^{n}\left(B \times \Omega_{X}\right) F(d x)=\left(\mathcal{I}^{* n} * F\right)\left(B \times \Omega_{X}\right)=E_{\infty}\left(B \times \Omega^{\infty}\right),
$$

where we have used Eq. (13) in the second equality. This implies that $E_{\infty}=\tilde{E}_{\infty} \leq F$, which completes the proof.

The part of the result of Theorem 4 (conservation of $E_{\infty}$ by $\mathcal{I}$ ) was first obtained in the Ph.D. thesis by the author. ${ }^{17}$

\section{EXAMPLES OF THE INFINITE COMPOSITION: PHOTON COUNTING AND QUANTUM COUNTER MEASUREMENTS}

In this section, we consider typical examples of standard Borel CP instruments, namely, photon counting ${ }^{1,3,18,19}$ and quantum counter ${ }^{3,8,20}$ instruments and evaluate the infinite compositions of them.

Let the system Hilbert space $\mathcal{H}$ correspond to a single-mode photon field, and have a complete orthonormal system $\{|n\rangle\}_{n \in \mathbb{N}}$ called photon number eigenstates. Here the set of natural numbers $\mathbb{N}$ contains 0 . We denote the power set of $\mathbb{N}$ as $2^{\mathbb{N}}$, and the countable product space of $\left(\mathbb{N}, 2^{\mathbb{N}}\right)$ as $\left(\mathbb{N}^{\infty}, \mathscr{B}\left(\mathbb{N}^{\infty}\right)\right)$.

The photon counting and quantum counter instruments for a finite time interval $t>0$ are discrete and pure $\mathrm{CP}$ instruments with an outcome space $\left(\mathbb{N}, 2^{\mathbb{N}}\right)$ defined by

$$
\begin{gathered}
\mathcal{I}_{B}^{\mathrm{pc}}(b):=\sum_{m \in B} M_{m}^{\mathrm{pc} *} b M_{m}^{\mathrm{pc}}, \\
M_{m}^{\mathrm{pc}}:=\sum_{n=0}^{\infty} \sqrt{p^{\mathrm{pc}}(m+n \mid n)}|n\rangle\langle n+m|, \\
p^{\mathrm{pc}}(m \mid n):=\left(\begin{array}{c}
n \\
m
\end{array}\right)\left(1-e^{-\lambda t}\right)^{m} e^{\lambda t(n-m)},
\end{gathered}
$$


for the photon counting instrument, ${ }^{1,3,18,21}$ and

$$
\begin{gathered}
\mathcal{I}_{B}^{\mathrm{qc}}(b):=\sum_{m \in B} M_{m}^{\mathrm{qc} *} b M_{m}^{\mathrm{qc}}, \\
M_{m}^{\mathrm{qc}}:=\sum_{n=0}^{\infty} \sqrt{p^{\mathrm{qc}}(m \mid n)}|n+m\rangle\langle n|, \\
p^{\mathrm{qc}}(m \mid n):=\left(\begin{array}{c}
n+m \\
m
\end{array}\right)\left(e^{\lambda t}-1\right)^{m} e^{-\lambda t(n+m+1),}
\end{gathered}
$$

for the quantum counter instrument. ${ }^{3,8}$ Here $\lambda$ is a positive constant corresponding to the coupling strength between the detector and the photon field. The infinite composition of $\mathcal{I}^{\text {pc,qc }}$ is a POVM $E_{\infty}^{\mathrm{pc}, \mathrm{qc}}$ with the infinite product outcome space $\left(\mathbb{N}^{\infty}, \mathscr{B}\left(\mathbb{N}^{\infty}\right)\right)$. Abusing the notation, $\mathcal{I}_{\{m\}}^{\mathrm{pc}, \mathrm{qc}}(\cdot)$ is denoted as $\mathcal{I}_{m}^{\mathrm{pc}, \mathrm{qc}}(\cdot)$.

We define the photon number observable $E^{N}(\cdot)$ by

$$
E^{N}(B):=\sum_{n \in B}|n\rangle\langle n| \quad\left(B \in 2^{\mathbb{N}}\right)
$$

and a POVM $E^{X}$ with its outcome space $\left(\mathbb{R}_{+}, \mathscr{B}\left(\mathbb{R}_{+}\right)\right)$, where $\mathbb{R}_{+}$is a real half-line $(0, \infty)$ and $\mathscr{B}\left(\mathbb{R}_{+}\right)$ is the Borel $\sigma$-algebra of $\mathbb{R}_{+}$, by

$$
\begin{gathered}
E^{X}(B)=\int_{B} F_{x} d x \quad\left(B \in \mathscr{B}\left(\mathbb{R}_{+}\right)\right), \\
F_{x}=\sum_{n \in \mathbb{N}} \frac{e^{-x} x^{n}}{n !}|n\rangle\langle n| .
\end{gathered}
$$

Here, $d x$ in Eq. (18) is the ordinary Borel measure on the real line.

The following theorem gives explicit forms of the infinite compositions of $\mathcal{I}^{\mathrm{pc}, \mathrm{qc}}$.

\section{Theorem 5.}

1. $E_{\infty}^{\mathrm{pc}} \simeq E^{N}$,

2. $E_{\infty}^{\mathrm{qc}} \simeq E^{X}$.

Proof.

1. From

$$
\begin{aligned}
\mathcal{I}_{m}^{\mathrm{pc}}\left(\left|n_{1}\right\rangle\left\langle n_{1}\right|\right) & =p^{\mathrm{pc}}\left(m \mid m+n_{1}\right)\left|m+n_{1}\right\rangle\left\langle m+n_{1}\right| \\
& =\sum_{n \in \mathbb{N}} \delta_{n, m+n_{1}} p^{\mathrm{pc}}(m \mid n)|n\rangle\langle n|
\end{aligned}
$$

and

$$
\begin{aligned}
\sum_{m, n_{1} \in \mathbb{N}} \delta_{n, m+n_{1}} \mathcal{I}_{m}^{\mathrm{pc}}\left(\left|n_{1}\right\rangle\left\langle n_{1}\right|\right) & =\sum_{m, n_{1} \in \mathbb{N}} \delta_{n, m+n_{1}} p^{\mathrm{pc}}\left(m \mid m+n_{1}\right)\left|m+n_{1}\right\rangle\left\langle m+n_{1}\right| \\
& =|n\rangle\langle n|,
\end{aligned}
$$

we obtain $\mathcal{I}^{\mathrm{pc}} * E^{N} \simeq E^{N}$. Thus, from Theorem $4, E_{\infty}^{\mathrm{pc}} \leq E^{N}$ holds.

In order to show $E^{N} \leq E_{\infty}^{\mathrm{pc}}$, let us define $\mathscr{B}\left(\mathbb{N}^{\infty}\right)$-measurable stochastic variables $M_{k}\left(\boldsymbol{m}^{(\infty)}\right)$ and $M_{\infty}\left(\boldsymbol{m}^{(\infty)}\right)$ by

$$
\begin{gathered}
M_{k}\left(\boldsymbol{m}^{(\infty)}\right):=\sum_{i=1}^{k} m_{k} \in \mathbb{N}, \\
M_{\infty}\left(\boldsymbol{m}^{(\infty)}\right):=\lim _{k \rightarrow \infty} M_{k}\left(\boldsymbol{m}^{(\infty)}\right) \in \mathbb{N} \cup\{\infty\},
\end{gathered}
$$

where $\boldsymbol{m}^{(\infty)}:=\left(m_{1}, m_{2}, \ldots\right) \in \mathbb{N}^{\infty}$. Since $\delta_{m, M_{k}\left(\boldsymbol{m}^{(\infty)}\right)} \rightarrow \delta_{m, M_{\infty}\left(\boldsymbol{m}^{(\infty)}\right)}$ for each $m \in \mathbb{N}$ and each $\boldsymbol{m}^{(\infty)} \in \mathbb{N}^{\infty}$, we have 


$$
\begin{aligned}
E^{M_{\infty}}(m) & :=\int_{\mathbb{N}^{\infty}} \delta_{m, M_{\infty}\left(\boldsymbol{m}^{(\infty)}\right)} E_{\infty}^{\mathrm{pc}}\left(d \boldsymbol{m}^{(\infty)}\right) \\
& =\lim _{k \rightarrow \infty} \int_{\mathbb{N}^{\infty}} \delta_{m, M_{k}\left(\boldsymbol{m}^{(\infty)}\right)} E_{\infty}^{\mathrm{pc}}\left(d \boldsymbol{m}^{(\infty)}\right) \\
& =\lim _{k \rightarrow \infty} \sum_{m_{1}, \ldots, m_{k} \in \mathbb{N}} \delta_{m_{1}+\cdots+m_{k}, m} \mathcal{I}_{m_{1}}^{\mathrm{pc}} \circ \cdots \circ \mathcal{I}_{m_{k}}^{\mathrm{pc}}(I) .
\end{aligned}
$$

Here $E^{M_{\infty}}(m)=E^{M_{\infty}}(\{m\})$ is the POVM derived by $M_{\infty}$ and the limit is in the sense of the weak operator topology. Let us evaluate Eq. (21). From Eq. (19), for each $k \geq 1$ and $\left(m_{1}, \ldots, m_{k}, n_{k}\right) \in \mathbb{N}^{k+1}$ we have

$$
\begin{aligned}
& \mathcal{I}_{m_{1}}^{\mathrm{pc}} \circ \cdots \circ \mathcal{I}_{m_{k}}^{\mathrm{pc}}\left(\left|n_{k}\right\rangle\left\langle n_{k}\right|\right) \\
& =\left(\prod_{i=1}^{k} p^{\mathrm{pc}}\left(m_{i} \mid m_{i}+\cdots+m_{k}+n_{k}\right)\right)\left|m_{1}+\cdots+m_{k}+n_{k}\right\rangle\left\langle m_{1}+\cdots+m_{k}+n_{k}\right|,
\end{aligned}
$$

and thus

$$
\begin{aligned}
& \mathcal{I}_{m_{1}}^{\mathrm{pc}} \circ \cdots \circ \mathcal{I}_{m_{k}}^{\mathrm{pc}}(I) \\
& =\sum_{m_{1}, \ldots, m_{k}, n \in \mathbb{N}} p_{k}^{\mathrm{pc}}\left(m_{1}, \ldots, m_{k} \mid n\right)|n\rangle\langle n|,
\end{aligned}
$$

where

$$
p_{k}^{\mathrm{pc}}\left(m_{1}, \ldots, m_{k} \mid n\right):=\prod_{i=1}^{k} p^{\mathrm{pc}}\left(m_{i} \mid n-m_{1}-\cdots-m_{i-1}\right) .
$$

By performing some calculations, the distribution of $m_{1}+\cdots+m_{k}$ for conditional distribution Eq. (22) is evaluated to be

$$
\begin{aligned}
p_{k}^{\mathrm{pc}}(m \mid n) & :=\sum_{m_{1}, \ldots, m_{k} \in \mathbb{N}} \delta_{m_{1}+\cdots+m_{k}, m} p_{k}^{\mathrm{pc}}\left(m_{1}, \ldots, m_{k} \mid n\right) \\
& =\left(\begin{array}{c}
n \\
m
\end{array}\right)\left(1-e^{-\lambda t k}\right)^{m}\left(e^{-\lambda t k}\right)^{n-m} \\
& \rightarrow \delta_{n, m} \quad(k \rightarrow \infty) .
\end{aligned}
$$

Thus, from Eq. (21), we have

$$
E^{M_{\infty}}(m)=\lim _{k \rightarrow \infty} \sum_{n \in \mathbb{N}} p_{k}^{\mathrm{pc}}(m \mid n)|n\rangle\langle n|=| m\rangle\langle m|,
$$

which implies that $E^{N}=E^{M_{\infty}} \leq E_{\infty}^{\mathrm{pc}}$ and we have proved the assertion. Note that Eq. (23) indicates that $E^{M_{\infty}}(\infty)=I-E^{M_{\infty}}(\mathbb{N})=O$, i.e., $M_{k}$ is convergent $E_{\infty}^{\mathrm{pc}}$-almost surely.

2. From

$$
\begin{gathered}
\mathcal{I}_{m}^{\mathrm{qc}}\left(F_{x}\right)=e^{-\lambda t} p^{\mathrm{qc}}\left(m \mid e^{-\lambda t} x\right) F_{e^{-\lambda t} x}, \\
p^{\mathrm{qc}}(m \mid x):=\frac{\left[\left(e^{\lambda t}-1\right) x\right]^{m}}{m !} \exp \left[-\left[\left(e^{\lambda t}-1\right) x\right]\right],
\end{gathered}
$$

we have

$$
\begin{aligned}
\mathcal{I}_{m_{1}}^{\mathrm{qc}} \circ \cdots \circ \mathcal{I}_{m_{k}}^{\mathrm{qc}}\left(F_{x}\right) & =e^{-\lambda t k}\left(\prod_{i=1}^{k} p^{\mathrm{qc}}\left(m_{i} \mid e^{-\lambda t(k-i+1)} x\right)\right) F_{e^{-\lambda t k_{x}}}, \\
\mathcal{I}_{m_{1}}^{\mathrm{qc}} \circ \cdots \circ \mathcal{I}_{m_{k}}^{\mathrm{qc}}(I) & =\int_{0}^{\infty} \mathcal{I}_{m_{1}}^{\mathrm{qc}} \circ \cdots \circ \mathcal{I}_{m_{k}}^{\mathrm{qc}}\left(F_{x}\right) d x \\
& =\int_{0}^{\infty}\left(\prod_{i=1}^{k} p^{\mathrm{qc}}\left(m_{i} \mid e^{\lambda t(i-1)} x\right)\right) E^{X}(d x) .
\end{aligned}
$$


Let $v_{x}^{\infty}(\cdot)$ be the product measure of $p^{\mathrm{qc}}\left(\cdot \mid e^{\lambda t(i-1)} x\right)$ with respect to $i \geq 1$ with its outcome space $\left(\mathbb{N}^{\infty}, \mathscr{B}\left(\mathbb{N}^{\infty}\right)\right)$. Then Eq. (24) implies that

$$
E_{\infty}^{\mathrm{qc}}(\cdot)=\int_{0}^{\infty} v_{x}^{\infty}(\cdot) E^{X}(d x),
$$

and we have shown $E_{\infty}^{\mathrm{qc}} \leq E^{X}$. To show $E^{X} \leq E_{\infty}^{\mathrm{qc}}$, let us define $\mathscr{B}\left(\mathbb{N}^{\infty}\right)$-measurable stochastic non-negative variables $X_{k}\left(\boldsymbol{m}^{(\infty)}\right)$ by

$$
X_{k}\left(\boldsymbol{m}^{(\infty)}\right):=e^{-\lambda t k} \sum_{i=1}^{k} m_{i} .
$$

If we denote the expectation with respect to $v_{x}^{\infty}(\cdot)$ as $\mathbb{E}_{x}[\cdot]$, we have

$$
\begin{gathered}
\mathbb{E}_{x}\left[X_{k}\right]=\left(1-e^{-\lambda t k}\right) x, \\
\mathbb{E}_{x}\left[\left(X_{k}-\mathbb{E}_{x}\left[X_{k}\right]\right)^{2}\right]=e^{-\lambda t k}\left(1-e^{-\lambda t k}\right) x,
\end{gathered}
$$

where we have omitted the dependence of $\boldsymbol{m}^{(\infty)} \in \mathbb{N}^{\infty}$. Thus, from Chebyshev's inequality we obtain

$$
v_{x}\left(\left\{\left|X_{k}-x\right|>e^{-\lambda t k / 4}\right\}\right) \leq e^{\lambda t k / 2} \mathbb{E}_{x}\left[\left|X_{k}-x\right|^{2}\right] \leq C_{x} e^{-\lambda t k / 2},
$$

where $C_{x}$ is some positive constant independent of $k$. Then Borel-Cantelli lemma assures that $X_{k}$ converges to $x v_{x}^{\infty}$-almost surely. Therefore, from Eq. (25), $X_{k}$ is convergent $E_{\infty}^{\mathrm{qc}}$-almost surely. Then, we can define a non-negative stochastic variable $X_{\infty}:=\lim _{k \rightarrow \infty} X_{k}$, which satisfies $X_{\infty}=x v_{x}^{\infty}$-almost surely, i.e., $v_{x}\left(X_{\infty}^{-1}(B)\right)=\chi_{B}(x)$ for each $B \in \mathscr{B}_{(}\left(\mathbb{R}_{+}\right)$. Therefore, for each $B \in \mathscr{B}\left(\mathbb{R}_{+}\right)$, we have

$$
\begin{aligned}
\int_{\mathbb{N}^{\infty}} \chi_{B}\left(X_{\infty}\left(\boldsymbol{m}^{(\infty)}\right)\right) E_{\infty}^{\mathrm{qc}}\left(d \boldsymbol{m}^{(\infty)}\right) & =E_{\infty}^{\mathrm{qc}}\left(X_{\infty}^{-1}(B)\right) \\
& =\int_{0}^{\infty} v_{x}^{\infty}\left(X_{\infty}^{-1}(B)\right) E^{X}(d x) \\
& =\int_{0}^{\infty} \chi_{B}(x) E^{X}(d x)=E^{X}(B),
\end{aligned}
$$

which implies $E^{X} \leq E_{\infty}^{\mathrm{qc}}$. Thus, we have proved $E_{\infty}^{\mathrm{qc}} \simeq E^{X}$.

According to the above proof, we also obtain the following theorem concerning the convergences of the stochastic variables $M_{k}$ and $X_{k}$.

\section{Theorem 6.}

(i) $M_{k}$ defined by Eq. (20) is convergent $E_{\infty}^{\mathrm{pc}}$-almost surely and the POVM corresponding to the distribution of $\lim _{k \rightarrow \infty} M_{k}$ coincides with the photon number observable $E^{N}$.

(ii) $X_{k}$ defined by Eq. (26) is convergent $E_{\infty}^{\mathrm{qc}}$-almost surely and the POVM corresponding to the distribution of $\lim _{k \rightarrow \infty} X_{k}$ coincides with $E^{X}$.

We remark that the statement of Theorem 6 (ii) is essentially the same as Theorem 4 of Ref. 8 while the proof of Ref. 8 is, rigorously speaking, insufficient due to the following reason. The authors of Ref. 8 show that the characteristic function of $X_{k}$ converges to that of $E^{X}$ and conclude the assertion of the theorem. However, as well-known in the measure theoretic probability theory, the pointwise convergence of the characteristic function, which is equivalent to the convergence in distribution, does not necessarily imply the almost sure convergence of a stochastic variable. In this sense, our proof of Theorem 6 (ii) complements the mathematically insufficient discussion of Ref. 8.

\section{SUMMARY}

In summary, we have rigorously reformulated the concept of the information conservation condition in Definition 1 depending on the equivalence relation among POVMs and the composition 
between an instrument and a POVM. By using quantum Kolmogorov extension theorem, we have constructed the infinite composition of a given standard Borel CP instrument $\mathcal{I}$. We have shown that the infinite composition is the least informative standard Borel POVM that is conserved by $\mathcal{I}$. We have considered specific examples of CP instruments, namely, photon counting and quantum counter instruments, and shown that their infinite compositions are equivalent to the photon number observable $E^{N}$ and the POVM $E^{X}$ given by Eq. (18), respectively. As a by-product of the proof, we have found some results on the almost sure convergences of the total counting number for the photon counting and the properly normalized counting number for the quantum counter cases, respectively. The latter result on the convergence in the quantum counter measurement complements the insufficiency of the proof in the existing work ${ }^{8}$ from the standpoint of the rigorous measure theoretic description of quantum measurements.

\section{ACKNOWLEDGMENTS}

The author acknowledges supports by Japan Society for the Promotion of Science (KAKENHI Grant No. 269905). He also would like to thank helpful discussions with Masahito Ueda (the University of Tokyo) and Tomohiro Shitara (the University of Tokyo).

${ }^{1}$ M. Srinivas and E. Davies, "Photon counting probabilities in quantum optics," Opt. Acta 28, 981-996 (1981).

${ }^{2}$ M. Ozawa, "Quantum measuring processes of continuous observables," J. Math. Phys. 25, 79-87 (1984).

${ }^{3}$ Y. Kuramochi and M. Ueda, "Classicality condition on a system observable in a quantum measurement and a relative-entropy conservation law,” Phys. Rev. A 91, 032110 (2015).

${ }^{4}$ S. Dorofeev and J. de Graaf, "Some maximality results for effect-valued measures," Indag. Math. (N.S.) 8, 349-369 (1997)

${ }^{5}$ W. M. de Muynck, Foundations of Quantum Mechanics, an Empiricist Approach (Kluwer Academic Publishers, Dordrecht, Boston, London, 2002).

${ }^{6}$ T. Heinonen, "Optimal measurements in quantum mechanics," Phys. Lett. A 346, 77-86 (2005).

${ }^{7}$ A. Jenčová, S. Pulmannová, and E. Vinceková, "Sharp and fuzzy observables on effect algebras," Int. J. Theor. Phys. 47, 125-148 (2008).

${ }^{8}$ M. Ueda, N. Imoto, and H. Nagaoka, "Logical reversibility in quantum measurement: General theory and specific examples," Phys. Rev. A 53, 3808-3817 (1996).

${ }^{9}$ M. Ban, "State reduction, information and entropy in quantum measurement processes," J. Phys. A: Math. Gen. 32, 1643 (1999).

${ }^{10}$ P. R. Halmos and L. J. Savage, "Application of the Radon-Nikodym theorem to the theory of sufficient statistics," Ann. Math. Stat. 20, 225-241 (1949).

${ }^{11}$ T. Heinosaari and M. Ziman, The Mathematical Language of Quantum Theory: From Uncertainty to Entanglement (Cambridge University Press, 2011).

12 E. B. Davies, Quantum Theory of Open Systems (IMA, 1976).

${ }^{13}$ E. B. Davies and J. Lewis, "An operational approach to quantum probability," Commun. Math. Phys. 17, 239-260 (1970).

${ }^{14}$ S. M. Srivastava, A Course on Borel Sets (Springer, 1998).

${ }^{15}$ R. Tumulka, "A Kolmogorov extension theorem for POVMs," Lett. Math. Phys. 84, 41-46 (2008).

16 A. N. Kolmogorov, Grundbegriffe der Wahrscheinlichkeitsrechnung (Springer, 1933).

${ }^{17}$ Y. Kuramochi, "Relative-entropy conservation law in quantum measurement and its applications to continuous measurements," Ph.D. thesis, Department of Physics, The University of Tokyo, 2015, to be published in 2016 in http://repository. dl.itc.u-tokyo.ac.jp/index_e.html.

${ }^{18}$ M. Ueda, "Probability-density-functional description of quantum photodetection processes," Quantum Opt.: J. Eur. Opt. Soc. Part B 1, 131 (1989).

${ }^{19}$ N. Imoto, M. Ueda, and T. Ogawa, "Microscopic theory of the continuous measurement of photon number," Phys. Rev. A 41, 4127-4130 (1990).

${ }^{20}$ M. Ueda and M. Kitagawa, "Reversibility in quantum measurement processes," Phys. Rev. Lett. 68, 3424-3427 (1992).

${ }^{21}$ M. Ueda, N. Imoto, and T. Ogawa, "Quantum theory for continuous photodetection processes," Phys. Rev. A 41, 3891-3904 (1990). 